\title{
NOTAS CACTOLOGICAS
}

Por Holia BRAVO H.

A fines de septiembre de 1950, recibimos en el Instituto de Biología de la Universidad Nacional Autónoma de México, un envío de cactáceas procedentes de Cananea, Sonora, colectadas por el señor Dudley B. Gold; algunas de estas plantas ya venían determinadas por él. Días. después nos trajo él mismo al Instituto una reseña de los lugares que visitó y la lista de las especies encontradas en cada región.

Después de hacer un estudio de las especies, pudimos apreciar que en su mayoría son ya conocidas y figuran en la sistemática, con excepción, posiblemente, de una Marmmillaria y una Coryphantha que tenemos en estudio; estas especies también se encuentran en Ärizona, pues la región explorada forma parte de la misma zona geográfico-botánica y vienen ya descritas en la obra "Arizona Cacti"., de que es autor Leman Benson.

La presente nota tiene por objeto señalar en México la presencia de algunas de estas especies.

A continuación daremos una reseña de los lugares que se exploraron y de las especies que allí se encontraron, según los datos proporcionados por el señor Gold.

Arroyo de Puertecitos, Cananea, Sonora:

Este lugar queda a unos 5 kilómetros al norte de Cananea, a una altura de $1.600 \mathrm{~m}$; el arroyo, en esta parte, corta la Mesa al pie de Ta sierra, corriendo de oeste a este y dejando la orilla norteña expuesta al sol. En esta partie colectaron: Opunila phaeacantha Engelmann.1 Opuntia engelmannil Salm-Dyck, Opuntia santa-xita (Griffiths Y Haho) Rose, Opuntia mecrorhize Engelmoinn, Echinocereus rigidissimus (Engelmann) Rose, Echinocereus Rendlen (Engelmann) Rümpler y Coryphantha recurvata (Engelmann) Britton y Rose. 
Opuntia santa-rita se encuentra en las peñas y Opuntia macrorhiza en el fondo del arroyo. Conviven con estas cactáceas: Fouquieria splen. dens Engelmann y algunas especies de Yucca, Agave, Salix, Alnus, Fraxinus, Junglans y Quercus, y en las lomas un Juniperus y Cupresus arizonica Greene.

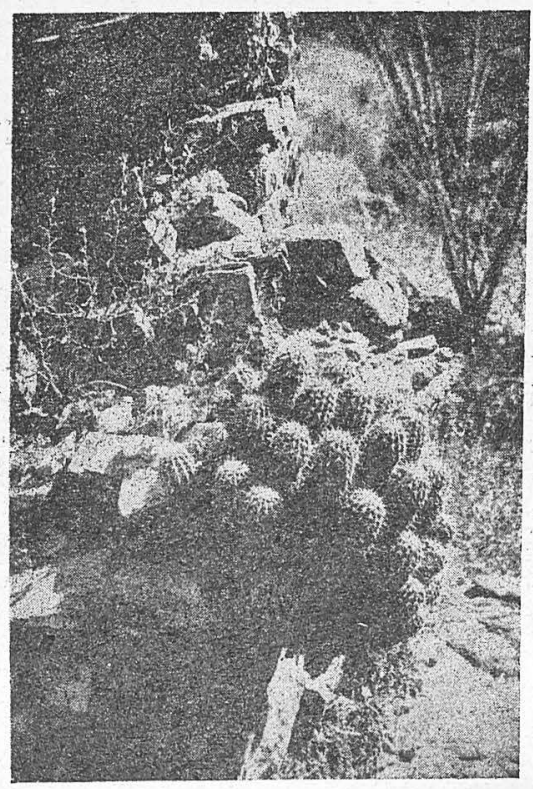

Echinocereus ledingii.

Cañón de Ymuris: Se encuentra al este de Ymuris y corta una se. rranía que separa el Valle de Ymuris del Valle de Cocospera. La altura varía entre 850 y $1.000 \mathrm{~m}$. El suelo es volcánico y es la zona bastante árida. Existen: "sahuaros" Cermegiea gigantea (Engelmann) Britton y Rose, en todo el Cañón y "pitahayos" Lemaireocereus trurberi (Engelmann) Britton y Rose en la parte más baja. Las especies colectadas fueron: Lemaireocereus thurberi (Engelmann) Britton y Rose, Mammillaria microcarpa Engelmann, Mammilleria macdougalii Rose y Mammin llaria hemispharerica Engelmann. Los géneros más conspicuos del fruticetum que acompaña a estas cactóceas son: Salix. Alnus, Prosopis y Celtis. Son escasas las especies de Opuntia y Cylindropuntia "choyas".

Mesa de Ymuris: Aqui existen bastantes "sahuaros" Carnegiea gi- 
gantea (Engelmann) Britton y Rose "pitahayos" Lemaireocereus thurberi (Engelmann( Britton y Rose, varias especies de choyas" y Echinocereus tendeleri (Engelmann) Briton y Rose. Caracteriza la associación de esta flora el "mezquite". Prosopis julitlora De Candolle.

Magdalena: La vegetación de Magdalena es parecida a la de Ymuris pues forma parte del mismo Valle. Al sureste hay una mesa poblada por "sahuaros". Se colectaron: Ferocactus covillei Britton y Rose y una Coryphantha que tenemos en estudio.

San Ignacio: Está entre Magdalena y Santa Ana. Hay abundantes "sahuaros" y Lophocereus schotti (Engelmann) Briton y Rose. Se colectó en este sitio Carnegiea gigantea (Engelmann) Britton y Rose.

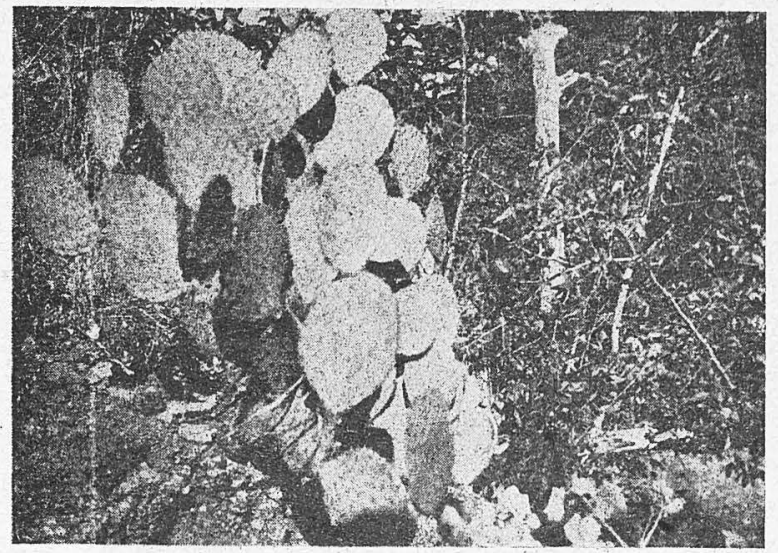

Opuntia Santa-rita

Santa Ana: Al oeste de Santa Ana empieza la zona desértica con vegetación notablemente xerófita. Determina la vegetación la "gobernadora" Larrea iridentata, y es común la "sinita" Lophocereus schotti (Engelmann) Britton y Rose.

A lo largo de la carretera, al sur de Santa Ana, se colectaron: Camegiea gigantea (Engelmann) Britton $Y$ Rose, Echinccereus fendleri (Engelmann) Britton y Rose var. robustus Peebles. Opuntia leptocaulis De Candolle,. Opuntia arbuscula Engelmann, Opuntia kleiniae De 
Candolle, Opuntia bigelowii Engelmann, Opuntia echinocarpa Engelmann * Bigelow, Opuntia thurberi Engelmann y una Mammillaria que estamos estudiando. Existe también en esta zon $\alpha$ Ratbunia alamosensis (Coulter) Britton y Rose. Como se puede notar esta es la zona de las "choyas". encontróndose cuando menos seis especies distintas.

Arroyo del Aserradero, Sierra de los Ajos. El señor Gold reconrió este arroyo primero desde el lugar donde cruza el camino de Cananea a Bacoachi (a $1.400 \mathrm{~m}$. de altura) hasta el punto donde sale la Sierra

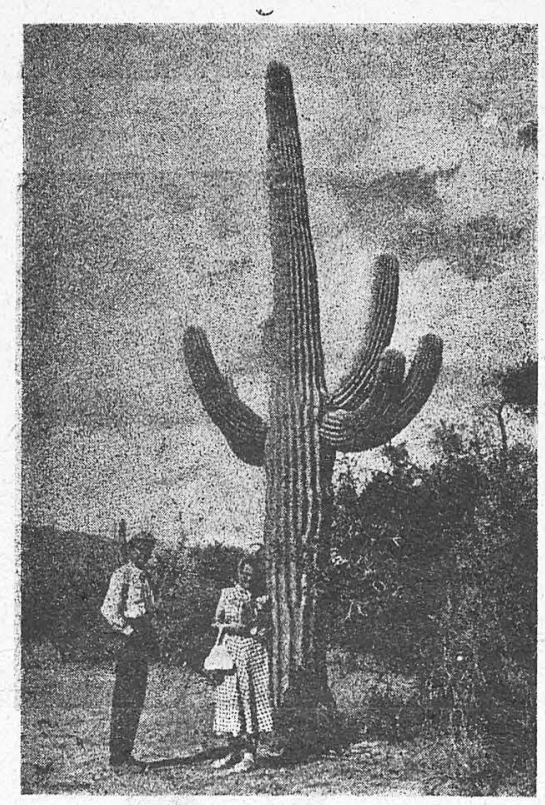

Cornegiea gigantea

de los Ajos (Campo Evans, a $1: 550 \mathrm{~m}$.), y después hasta el camino del aserradero, unos cinco kilómetros dentro de la Sierra, 1.700 m. Hasta el campo Evans el arroyo es ancho y abierto y está poblado de Quercus: desde el campo Evans para arriba la vegetáción es de clima trío: hay Acex, Alnus, Platanus y varias especies de Pinus. En esta parte 'fueron colectados Echinocereus ledingiil Peebles (fig. 4), Echinocereus Hered 
tton y Rose y Opuntia discaita Griffiths Echinocereus ledingii Peebles, es abundante en las peñas desde el Campo Evans hasta el camino del aserradero: el señor Gold también ha colectado esta especie en la Sierra Graham, en Arizona, a $.800 \mathrm{~m}$. de altura.

Camino de Cananea a Bacoachi (arroyo a $18 \mathrm{Km}$. de Cananea). Äquí se colectó Opuntia saniár-ríta (Griffiths y Hare) Rose, Echinocereus rigidissimus (Engelmann) Rose $Y$ Coryphantha recurvato (Engelmann) Britton y Rose. 